\title{
Evaluating Trajectory Queries Over Imprecise Location Data
}

\author{
Xike Xie $^{1 \star} \quad$ Reynold Cheng ${ }^{2} \quad$ Man Lung Yiu ${ }^{3}$ \\ 1 Aalborg University, Denmark \\ xkxiedcs.aau.dk \\ ${ }^{2}$ University of Hong Kong, Pokfulam Road, Hong Kong \\ ckcheng@cs.hku.hk \\ 3 Hong Kong Polytechnic University, Hung Hom, Hong Kong \\ csmlyiu@comp.polyu.edu.hk
}

\begin{abstract}
Trajectory queries, which retrieve nearby objects for every point of a given route, can be used to identify alerts of potential threats along a vessel route, or monitor the adjacent rescuers to a travel path. However, the locations of these objects (e.g., threats, succours) may not be precisely obtained due to hardware limitations of measuring devices, as well as the constantly-changing nature of the external environment. Ignoring data uncertainty can render low query quality, and cause undesirable consequences such as missing alerts of threats and poor response time in rescue operations. Also, the query is quite time-consuming, since all the points on the trajectory are considered. In this paper, we study how to efficiently evaluate trajectory queries over imprecise location data, by proposing a new concept called the $u$-bisector. In general, the $u$-bisector is an extension of bisector to handle imprecise data. Based on the $u$-bisector, we design several novel filters to make our solution scalable to a long trajectory and a large database size. An extensive experimental study on real datasets suggests that our proposal produces better results than traditional solutions that do not consider data imprecision.
\end{abstract}

\section{Introduction}

Given a set $P$ of points, the Trajectory Nearest Neighbor Query (TNNQ in short) [1], retrieves the closest object in $P$ for every query point on the given trajectory $T$. As an example, consider the trajectory $T=\left\{\left[q_{1}, q_{2}\right],\left[q_{2}, q_{3}\right],\left[q_{3}, q_{4}\right]\right\}$ and objects $P=$ $\left\{o_{1}, o_{2}, o_{3}\right\}$, shown in Figure 1 a). The TNNQ's answer is as Figure 1 (b). It means for all points on $\left[s_{0}^{\prime}, s_{1}^{\prime}\right]$, the nearest neighbor is $o_{1}$, etc. The $T N N Q$ can find applications in location-based service (LBS in short), such as "what is the nearest gas station along the travel route".

Unfortunately, the measured location of an object is often imprecise because of: (i) limited resolution of the measure device, (ii) infrequent measurement, (iii) environmental factors. For example, the shipping industries regard safety as their top priority. They hope to identify alerts of potential threats along the route of a vessel in advance, and take appropriate actions if necessary. People in the US and Northern Europe detect the

* This work is done in the University of Hong Kong. 
icebergs by remote sensors and satellite imaging [2], which have limited measurement accuracy and frequency. Sensors have limited battery capacity whereas satellite imaging incurs expensive deployment cost. This causes infrequent measurements, rendering the measured location of an object stale. Furthermore, as time passes by, icebergs may move according to the temperature and the ocean current / wind speed. In the LBS example, what if the objects being queried are not static but moving constantly (e.g. rescue vehicles positioned by GPS devices)? Again, locations obtained by GPS devices can be contaminated with measurement error, which can be further deteriorated by terrain and climate conditions [3]. Also, the positions could be tracked only periodically due to the limited battery powers [4].

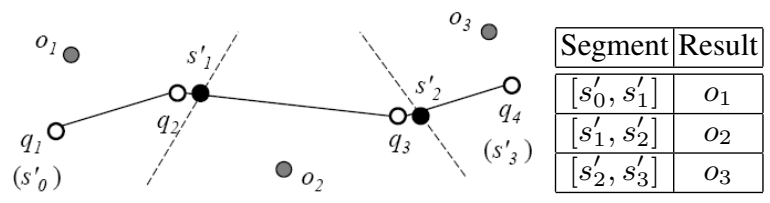

(a) precise objects

(b) result

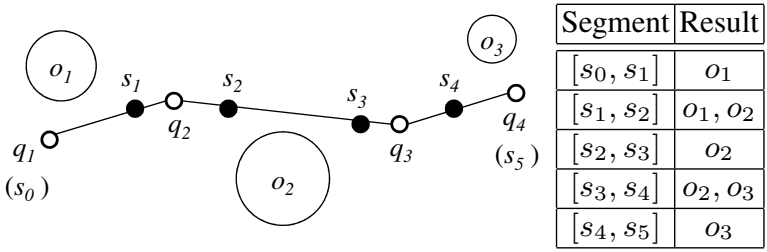

(c) imprecise objects

(d) result

Fig. 1. Example Trajectory Query

A common way to represent an imprecise location or a moving object is to model the position by an area called imprecise region [4|56778|9|10]. The possible location of the object is assumed to be within this region. Figure 1(c) shows a query trajectory $T=\left\{\left[q_{1}, q_{2}\right],\left[q_{2}, q_{3}\right],\left[q_{3}, q_{4}\right]\right\}$ and some imprecise objects $o_{1}, o_{2}, o_{3}$. The result (Figure 1(d)) can be represented in a compact way by partitioning the query trajectory into segments such that all locations within the same segment share the same result set. For example, $o_{2}$ is definite nearest neighbor to the segment $\left[s_{2}, s_{3}\right]$. On the other hand, $o_{1}$ and $\mathrm{o}_{2}$ are possible nearest neighbors (PNNs) to the segment $\left[s_{1}, s_{2}\right]$ because both of them have potential to be the closest object. We define this query as Trajectory Possible Nearest Neighbor Query (TPNNQ in short). Note that [1] is a special case of our problem, where the objects being queried are precise points.

Determining the TPNNQ answer can be technically challenging, since the imprecise regions are considered. A simple solution is to replace the imprecise region of each object with a center point (shown as a grey dot), as illustrated in the scenario in Figure 1(a) and (b). The result consists of three segments, each associated with the closest object. For instance, the closest object to location $q_{2}$ appears to be $o_{1}$ only. The object $\mathrm{O}_{2}$ is missing from the result. Recall from Figure 1 (c) and (d) that $\mathrm{O}_{2}$ also has possibility to become a closest object to location $q_{2}$. This "center simplification" approach causes undesirable consequences such as missing alerts of threats and poor response time in our applications. In the vessel/rescuer example, the ignorance of the imprecise 
region could cause potential danger. Thus, it is important to augment each threat with an imprecise region, in order to foresee the worst-case scenario. In the rescuer example, a rescue vehicle seemingly close to / far from the travel path may be actually far from / close to it. Thus, it would take longer time to respond. It is better to call up all rescuers likely to be the closest, in order to handle the emergency as soon as possible.

Another attempt to simplify the problem is to use a "sampling approach", which considers positions at every fixed length on the query trajectory, and compute the potential nearby objects at each position. However, if the sampling rate is high, it incurs a huge computation cost; on the other hand, a low sampling rate can result in many answers missing. Notice that a query trajectory consists of infinite number of possible locations, and it is not easy to determine the sampling rate. As shown in Figure 11 (c), the result set changes only at a few positions $\left(s_{1}, s_{2}, s_{3}, s_{4}\right)$. It is not clear how to determine the correct sampling rate to in order to get these answers. In fact, our experimental results show that replacing imprecise regions with points or sampling the trajectory cannot provide an accurate solution. Hence, we develop a solution that can accurately compute a trajectory query on imprecise objects.

The techniques of [1] cannot be readily applied to evaluate $T P N N Q$. [1]'s idea is to use the (perpendicular) bisectors of every pair of points to derive the query answer. For example, in Figure 1 (a), the point $s_{1}^{\prime}$ is the intersection between the query trajectory and the bisector of objects $o_{1}$ and $o_{2}$, which are shown as dashed lines. Similarly, $s_{2}^{\prime}$ is derived by $o_{2}$ and $o_{3}$ 's bisector. However, the bisector, which forms the basis of [1], is limited to precise points.

We extend the concept of "bisectors" to support imprecise objects, called $u$ bisector. Figure 2 illustrates the corresponding $u$-bisectors for circular and rectangular imprecise regions. From this figure, we can see that the $u$-bisector is not a straight line anymore. It becomes a pair of lines, which partition the domain space into three parts: (1) the left area, containing points $q$ where $O_{i}$ is absolutely closer to $q$ than $O_{j}$; (2) the right partition, consisting of points $q^{\prime}$ where $O_{j}$ is absolutely closer to $q^{\prime}$ than $O_{i}$; and (3) the middle part, having points $q^{\prime \prime}$ where both $O_{i}$ and $O_{j}$ can be the nearest to $q^{\prime \prime}$. We demonstrate how to use conceptually the intersection points of the query trajectory and the $u$-bisectors to answer a trajectory query.

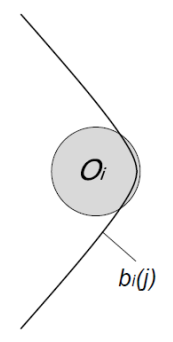

(a) circular imprecise region

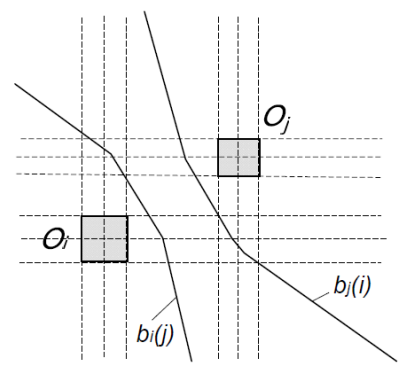

(b) rectangular imprecise region

Fig. 2. $u$-bisector for imprecise regions. 
In practice, it is expensive to compute the intersections points between the query trajectory and the $u$-bisectors. As shown in Figure 2, these $u$-bisectors can be hyperbolic curves (Figure 2(a)), or segments of straight lines/curves (Figure 2 b)). Even for one $u$-bisectors, they can intersect the query trajectory at more than one point. We first design a Basic solution, which answers the query in $O\left(\ln ^{2} \operatorname{logn}\right)$ (n:database size; l:trajectory length). To make our solution scalable to large datasets and long trajectories, we design a filter-refinement framework. In the filtering phase, candidate objects that may be the closest to each answer line-segment are obtained. In the refinement phase, we develop a novel technique called ternary decomposition, which can derive the final answers accurately. We show theoretically and experimentally that our solution is efficient and scalable. It is also more accurate than the center simplification and the sampling approaches. We assume the imprecise regions are of circular shapes for simplicity. Actually, our method is also general to other shaped objects. It would not be discussed due to page limitations.

The rest of this paper is as follows. In Section 2 we discuss the related work. Section 3 defines the problem and a basic solution based on the $u$-bisectors. We present our solution framework in Section 4 . The filtering and refinement phases are described in Sections 5 and 6 In Section 7 we present our experiment results. Section 8 concludes.

\section{Related Work}

Nearest neighbor (NN) query for moving query points is a well studied topic [11] [12] [13] [1]. These works focus on reducing the computational cost at the server. Among these works, there are two major categories.

The first category does not require the user's entire trajectory in advance [11] [12] [13], but processes the query online (multiple times) based on the user's moving location. In [11], the authors propose sampling techniques to answer the moving $N N$ query. They study how to calculate the upper-bound distance within which the moving point does not issue a new query to the server. [12] [13] use validity region and validity time for the query answer of moving points. They use Voronoi cells to represent the validity region. The query answer becomes invalid if the validity time is expired or the user leaves the validity region.

The second category assumes that the user's trajectory is known in advance. It evaluates the query once only [1] [4]. In our application, the trajectory, such as sailing routes, is known in advance. Thus, we elaborate the second category in details. In [1], the route of the query point is split into sub-line-segments, such that the $N N$ answer within the same sub-line-segment remains unchanged. A perpendicular bisector $\perp\left(p_{i}, p_{j}\right)$ between two points $p_{i}$ and $p_{j}$ is used to partition the trajectory query into two sub-trajectories, one being definitely closer to $p_{i}$ and the other being definitely closer to $p_{j}$. However, this technique is not applicable to our problem on imprecise location data. As shown in Figure 1, some segments like $\left[s_{1}, s_{2}\right]$ can have multiple $P N N \mathrm{~s}$ and it is challenging to derive them.

The bisector for imprecise objects has been addressed by a few works recently [5] [6] [7]. They use bisectors to determine the dominance relationship between objects. Our work is different because we consider a query trajectory, but not a query 
object. For the trajectory, our solution is capable of answering the query for every point it.

The paper [4] is closely related to our problem. It also uses an imprecise region to model the location of an object and compute the object closest to a given query segment. Unlike our work, [4] only computes the answer for segments with the definite nearest neighbor, such as $\left[s_{0}, s_{1}\right]$ in Figure 1 . It did not study how to compute objects that might be the closest, for some segment like $\left[s_{1}, s_{2}\right]$ in Figure 1 . Furthermore, their method scans the entire database to answer the query, thus it is not very scalable to data volumes.

\section{Problem and Preliminaries}

In this section, we describe the query semantics in Section 3.1. We introduce the $u$ bisector in Section 3.2 and propose a basic method in Section 3.3.

\subsection{Problem Setting}

We first introduce the definition of $P N N Q$ (studied in [14]), which is used to define $T P N N Q$, the query studied in this paper. Let $q$ be a point, and let $O_{i}$ be an imprecise object from an object set $O$. We use $\operatorname{dist}_{\min }\left(q, O_{i}\right)$ and $\operatorname{dist}_{\max }\left(q, O_{j}\right)$ to denote the minimum and maximum distances of object $O_{i}$ from $q$, respectively.

Definition 1. Possible Nearest Neighbor Query (PNNQ): Given a set of imprecise objects $O$ and a query point $q, O_{i} \in P N N Q(q)$, if $\nexists O_{j} \in O$, such that dist $\max _{\text {max }}\left(q, O_{j}\right)<$ dist $_{\min }\left(q, O_{i}\right)$.

In Figure 1 ( c), $P N N Q\left(q_{2}\right)=\left\{O_{1}, O_{2}\right\}$ implies that either $O_{1}$ or $O_{2}$ could be the $N N$ of the query point $q_{2}$. A query trajectory $\mathcal{T}$ can be represented by a set of query line-segments $\mathcal{T}=\left\{L_{1}, \ldots, L_{l}\right\}$, where $L_{i}$ is a query line-segment. For a query point $q$, whose trajectory is $\mathcal{T}$, the trajectory possible nearest neighbor query $(T P N N Q)$ returns $P N N$ s for all the points in $\mathcal{T}$. In other words, the query returns $\{\langle q, P N N Q(q)\rangle\}_{q \in \mathcal{T}}$. If the connected points on the trajectory have the same $P N N \mathrm{~s}$, we could merge them into a segment.

Definition 2. Trajectory Possible Nearest Neighbor Query (TPNNQ): Given a set of imprecise objects $O$ and a query trajectory $\mathcal{T}$, the answer for the TPNNQ query is a set of tuples $R=\left\{\left\langle T_{i}, R_{i}\right\rangle \mid T_{i} \subseteq \mathcal{T}, R_{i} \subseteq O\right\}$, where $P N N Q(q)=R_{i}\left(\forall q \in T_{i}\right)$.

In other words, the $T P N N Q$ splits $\mathcal{T}$ into a set of consecutive segments $\left\{T_{1}, T_{2}, \ldots, T_{t}\right\} . T_{i}$ is a sub-trajectory of $\mathcal{T}$. For $\forall q \in T_{i}, q$ has the same possible nearest neighbors $(P N N \mathrm{~s})$, then we call each $T_{i}$ a validity interval. The connection point of two consecutive segments, say $T_{i}$ and $T_{i+1}$, is called turning point, which indicates the change of $P N N Q$ answers. An example for a $T P N N Q$ over three imprecise objects $\left\{O_{1}, O_{2}, O_{3}\right\}$ is shown in Figure 1 (c). The trajectory query $\mathcal{T}(s, e)$ is split into 5 pieces of segments. Also, point $s_{1}$ is the turning point for $T\left(s_{0}, s_{1}\right)$ and $T\left(s_{1}, s_{2}\right)$.

Observe that there are two major differences between the results on imprecise objects and precise objects. Comparing Figures 1(c) and (a): (1) the imprecise case could 
have more tuples (5 compared to 3); (2) a query point in imprecise case might return a set of $P N N$ s instead of a single answer.

Thus, the TPNNQ can be answered by finding the turning points. Then, how to derive the turning points on a trajectory, given a set of imprecise objects? To address that, we first investigate the $u$-bisector, for imprecise objects. In general, the $u$-bisector splits the domain into several parts, such that query points on different parts could have different $P N N$ s. Then, the turning points can be evaluated by finding the intersections of the $u$-bisectors and the query trajectory. Next, we discuss the $u$-bisector.

\section{$3.2 u$-bisector}

Definition 3. Given two imprecise objects $O_{i}$ and $O_{j}$, their u-bisector consists of two lines: $b_{i}(j)$ and $b_{j}(i)$. The u-bisector half $b_{i}(j)$ is a set of points satisfying

$$
b_{i}(j)=\left\{z: \operatorname{dist}_{\max }\left(z, O_{i}\right)=\operatorname{dist}_{\min }\left(z, O_{j}\right)\right\}
$$

The curve $b_{i}(j)$ splits the domain into two half-spaces: $H_{i}(j)$ and $H_{i}(j)$, where $H_{i}(j)$ is the half closer to $O_{i}$ and $\overline{H_{i}(j)}$ is the complementary half.An example is shown in Figure 3. Thus we have:

$$
\begin{aligned}
& H_{i}(j)=\left\{z: \operatorname{dist}_{\max }\left(z, O_{i}\right) \leq \operatorname{dist}_{\min }\left(z, O_{j}\right)\right\} \\
& \overline{H_{i}(j)}=\left\{z: \operatorname{dist}_{\max }\left(z, O_{i}\right)>\operatorname{dist}_{\min }\left(z, O_{j}\right)\right\}
\end{aligned}
$$

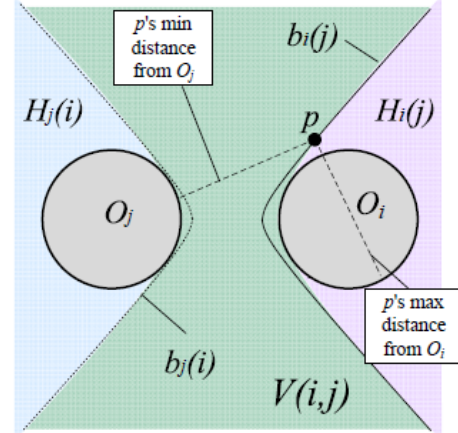

Fig. 3. $u$-bisector

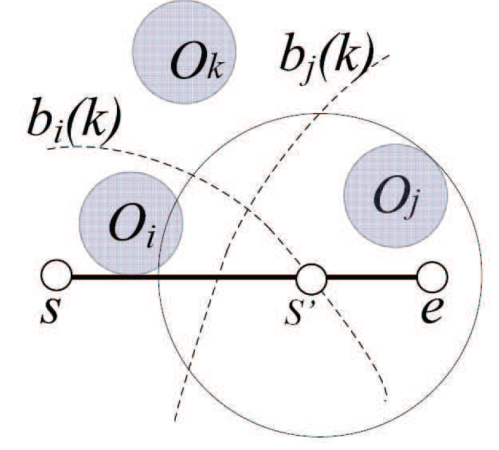

Fig. 4. Verification

Generally speaking, the $u$-bisector half $b_{i}(j)$ is a curve in the domain space. If a query point $q \in H_{i}(j), q$ must take $O_{i}$ as its nearest neighbor certainly. The $u$-bisector halves $b_{i}(j)$ and $b_{j}(i)$ separate the domain into three parts: $H_{i}(j), H_{j}(i)$, and $V(i, j)$, where

$$
V(i, j)=\overline{H_{i}(j)} \cap \overline{H_{j}(i)}
$$

Notice that $V(i, j)=V(j, i)$. If $O_{i}$ and $O_{j}$ are degenerated into precise points, $V(i, j)$ becomes 0 . Also, $b_{i}(j)=b_{j}(i)$, which becomes a straight line.

If a query line-segment is totally covered by $V(i, j)$ or $H_{i}(j)$, it does not intersect with $b_{i}(j)$. Otherwise, the intersections split the line-segment into several parts. Different parts might correspond to different $P N N \mathrm{~s}$, as they are located on different sides of $b_{i}(j)$. 
For circular imprecise objects, it is easy to derive the closed form equations of the $u$-bisector and evaluate the analytical solution for the intersection points. The number of intersections is at most 2 , since the quadratic equation has at most 2 roots (see Appendix A.1 . Next, we present the basic method based on the analysis of the $u$-bisector's intersections. We focus our discussion on two dimension location data.

\subsection{Basic Method}

From Definition 2, the TPNNQ could be answered by deriving the turning points, which are intersections of the query trajectory and the $u$-bisectors. A $u$-bisector is constructed by a pair of objects. Given a set $O$ of n objects, there can be $C_{2}^{n} u$-bisectors. The Basic method is to check the intersections of the query trajectory with the $C_{2}^{n} u$-bisectors. The intersections can be found by evaluating the equation's roots in Appendix A.1. Here we use FindIntersection(.) (in Step 5) to represent the process.

However, not all of the bisectors intersect with the trajectory. Even if they intersect, not all of the intersections are qualified as turning points. Thus, we need a "verification" process to exclude those unqualified intersectinos. For example, in Figure 4 the $u$ bisector half $b_{i}(k)$ intersect with $[s, e]$ at $s^{\prime}$. For an arbitrary point $q \in[s, e]$, either $O_{i}$ or $O_{j}$ is closer to $q$ than $O_{k}$, since $\left[s, s^{\prime}\right] \in H_{i}(k)$ and $\left[s^{\prime}, e\right] \in H_{j}(k)$. Then, $O_{k}$ is not $P N N$ for $p \in[s, e]$, and $s^{\prime}$ is not a qualified turning point.

We use the $s_{i \vdash j}$ to represent an intersection created by $b_{i}(j)\left(s_{i \vdash j}=b_{i}(j) \cap L\right)$, and $s_{i \dashv j}=b_{j}(i) \cap L$. In other words, $s_{i \vdash j}$ can be understood as $P N N Q(q)$ answer that turns from containing $O_{i}$ to both $O_{i}$ and $O_{j}$, if $q$ moves from $H_{i}(j)$ to $\overline{H_{i}(j)}$ So, $O_{i}$ should definitely be $s_{i \vdash j}$ 's $P N N$, while $O_{j}$ is not. This can be implemented by issuing a $P N N Q$. Thus, we can use this for verification.

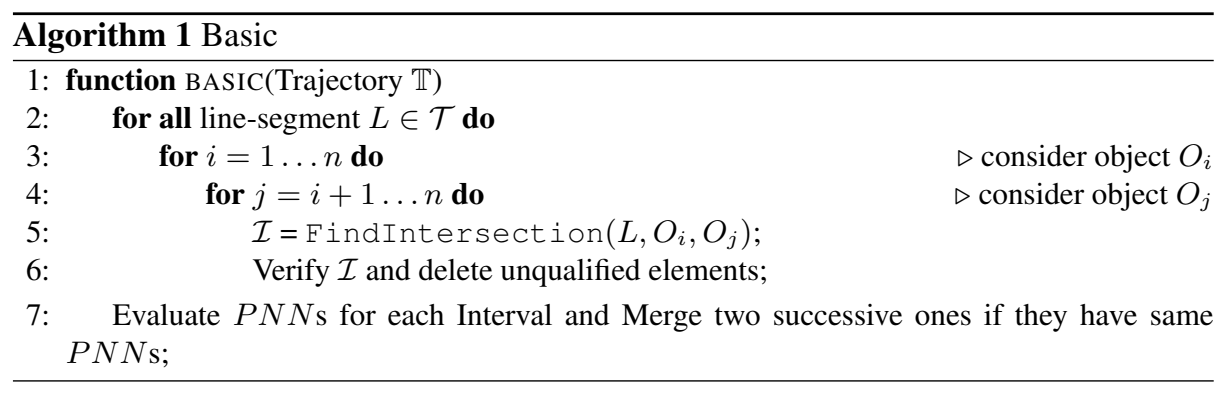

In Algorithm 1, suppose Step 5 can be done in time $\beta$, which is a constant if we call Appendix A.1 Step 6 can be finished in $O(\log n)$. Suppose $\mathcal{T}$ contains $l$ line-segments, Basic's total query time is $O\left(l n^{2}(\log n+\beta)\right)$. In later sections, we study several filters which can effectively prune those unqualified objects, which cannot be $P N N$ for any point on the trajectory, in order to reduce the complexity.

\section{Solution Framework}

In this section, we propose the framework of the $T P N N Q$ algorithm, which follows the filter-refinement framework. We assume an $\mathrm{R}$-tree $\mathbb{R}$ is built on the imprecise objects 
$O$ and it can be stored in the main memory, as the storage capabilities increase fast in recent years.

Framework In implementation, we organize the trajectory $\mathcal{T}=\left\{L_{1}, L_{2}, \ldots, L_{l}\right\}$ by constructing a binary tree $\mathbb{T}(\mathcal{T})$. Each binary tree node $T_{i}=\left\{L_{1}, \ldots, L_{l^{\prime}}\right\}$ has two children: $T_{i}$.left $=\left\{L_{1}, \ldots, L_{\left\lfloor\frac{l^{\prime}}{2}\right\rfloor}\right\}$ and $T_{i}$.right $=\left\{L_{\left\lceil\frac{l^{\prime}}{2}\right\rceil}, \ldots, L_{l^{\prime}}\right\}$. We show an example of $\mathcal{T}=\left\{L_{1}, L_{2}, L_{3}\right\}$ 's trajectory tree in Figure 5 (a).

The data structure for each binary tree node $T_{i}$ is a triple: $T_{i}=\langle L, M B C$, Guard $\rangle$. $L$ is a line-segment if $T_{i}$ is a leaf-node and $N U L L$ otherwise. $M B C$ is the minimum bounded circle covering $T_{i}$; it is $N U L L$ for leaf-nodes. Guard is an entry which has minimum maximum distance to $T_{i}$. As we describe later, the entry can be either an R-tree node or an imprecise object. The Guards are not initialized until the processing of $T P N N Q$. Since $\mathcal{T}$ contains $l$ line-segments, the trajectory tree $\mathbb{T}(\mathcal{T})$ could be constructed in $O(l \log l)$ time.

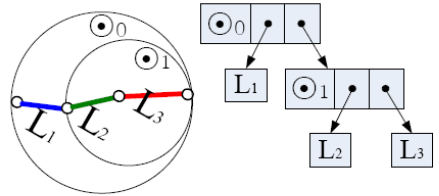

(a) Trajectory Tree $\mathbb{T}$

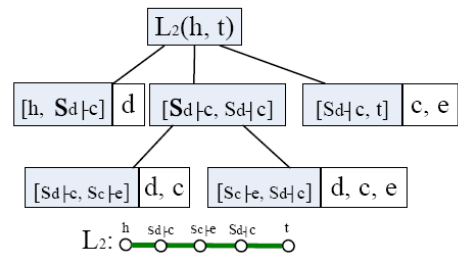

(b) Ternary Tree $\Psi\left(\mathrm{L}_{2}\right)$

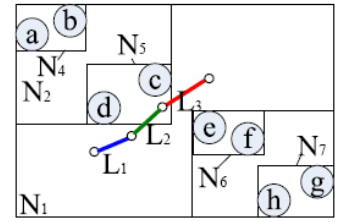

(a) Query Input

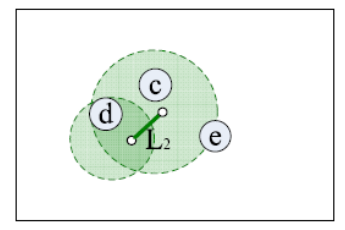

(c) Segment Filter $\mathrm{L}_{2}$

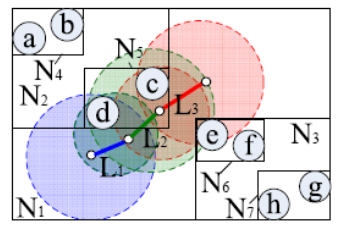

(b) Trajectory Filter

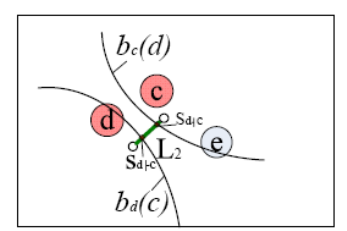

(d) Trajectory Refine $\mathrm{L}_{2}$

Fig. 5. Trajectory Tree $\mathbb{T}(\mathcal{T})$ and

Fig. 6. TPNNQ Ternary Tree $\Psi\left(L_{2}\right)$

Given a constructed trajectory tree $\mathbb{T}$ and an R-tree $\mathbb{R}$, the $T P N N Q$ algorithm, shown in Algorithm 2, consists of two phases. Phase I is the filtering phase, which includes two filters: Trajectory Filter and Segment Filter. Trajectory Filter is to retrieve a set of candidates from the database (Step 3). Segment Filter prunes away unqualified objects for each $L_{i} \in \mathcal{T}$ (Step 4). Phase II is to evaluate all the validity intervals and turning points for each line-segment of the trajectory (Step 5). Then, we scan the derived validity intervals once and merge two consecutive validity intervals if they belong to different line-segments but have the same set of PNNs (Step 7).

Example of TPNNQ Suppose an R-tree built on objects $O=\{a, b, c, d, e, f\}$ and a trajectory $\mathcal{T}=\left\{L_{1}, L_{2}, L_{3}\right\}$, as shown in Figure 6(a). We use Trajectory Filter to derive $\mathcal{T}$ 's trajectory filtering bound, as shown by shaded areas in Figure 6(b). The objects $\{c, d, e, f\}$ overlapping with the trajectory filtering bound are taken as candidates. During the process, object $d$ is set to be $L_{2}$ 's Guard, and stored in the trajectory tree. 


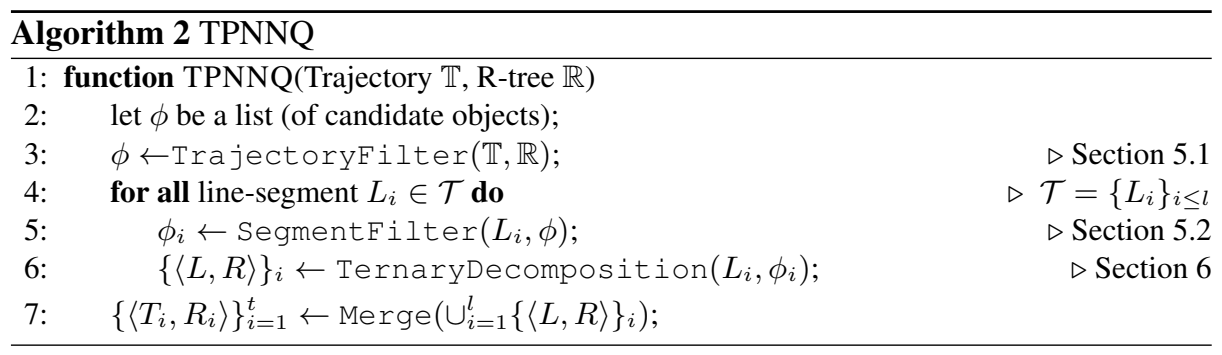

The segment filter is applied for each line-segment in $\mathcal{T}$. Taking $L_{2}(h, t)$ as an example, the segment filtering bound is shown as Figure 6(c), where $f$ is excluded from $L_{2}$ 's candidates. Because $f$ does not overlap with the filter bound.

In the refinement phase, we call the routine TernaryDecomposition to derive the turning points. We find the $u$-bisector halves $b_{d}(c)$ and $b_{c}(d)$ intersects with $L_{2}$ at $s_{d \vdash c}$ and $s_{d \dashv c}$, respectively. $L_{2}$ is split into three sub-line-segments $\left[h, s_{d \vdash c}\right],\left[s_{d \vdash c}, s_{d \dashv c}\right]$, and $\left[s_{d \dashv_{c}}, t\right]$. Meanwhile, the construction of a ternary tree $\Psi\left(L_{2}\right)$ starts, in Figure 5 (b). The root node of $\Psi\left(L_{2}\right)$ derives three children correspondingly.

Then, we repeat the above process for each of the three, recursively. Finally, the process stops and we get a ternary tree $\Psi\left(L_{2}\right)$, in Figure 5 (b). Observed from $\Psi\left(L_{2}\right)$, the degree of a ternary tree node is at most 3 , since a line-segment is split into at most 3 sub-line-segments, as shown in Section 3 The query result on $L_{2}$ can be fetched by traversing the leaf-nodes of $\Psi\left(L_{2}\right)$. Then, we have: TPNNQ $\left(L_{2}\right)=$ $\left\{\left\langle\left[h, s_{d \vdash c}\right],\{d\}\right\rangle,\left\langle\left[s_{d \vdash c}, s_{c \vdash e}\right],\{c, d\}\right\rangle,\left\langle\left[s_{c \vdash e}, s_{d \dashv c}\right],\{c, d, e\}\right\rangle,\left\langle\left[s_{d \dashv c}, t\right],\{c, e\}\right\rangle\right\}$. Similarly, the results of $L_{1}$ and $L_{3}$ can also be evaluated. By merging them we get the answer of $T P N N Q(\mathcal{T})$. After we get the query answer, $\mathbb{T}$ and $\Psi$ are deleted.

In the following sections, we study the Trajectory Filter in Section 5.1 and Segment Filter in Section 5.2. The refinement step is shown in Section 6

\section{Filtering Phase}

The trajectory query consists of a set of consecutive query line-segments. An intuitive way is to: (1) decompose the trajectory into several line-segments; (2) for each line-segment $L_{i}$, access R-tree to fetch candidates. Then, apply TernaryDecomposition to construct a ternary tree $\Psi\left(L_{i}\right)$ to evaluate its validity intervals and turning points. We call this method TP-S, which incurs multiple R-tree traversals.

Meanwhile, two consecutive line-segments might share similar $P N N s$. Also, if two line-segments are short, they could even be located within the same validity interval. So, considerable efficiency would be saved if the R-tree traversal for each line-segment inside the trajectory could be shared.

\subsection{Trajectory Filter}

To save the number of R-tree node access, we design Algorithm 3 as the Trajectory Filter to retrieve the candidates for the entire trajectory. We start Algorithm 3 by maintaining a heap in the ascending order of maximum distance between an entry to 


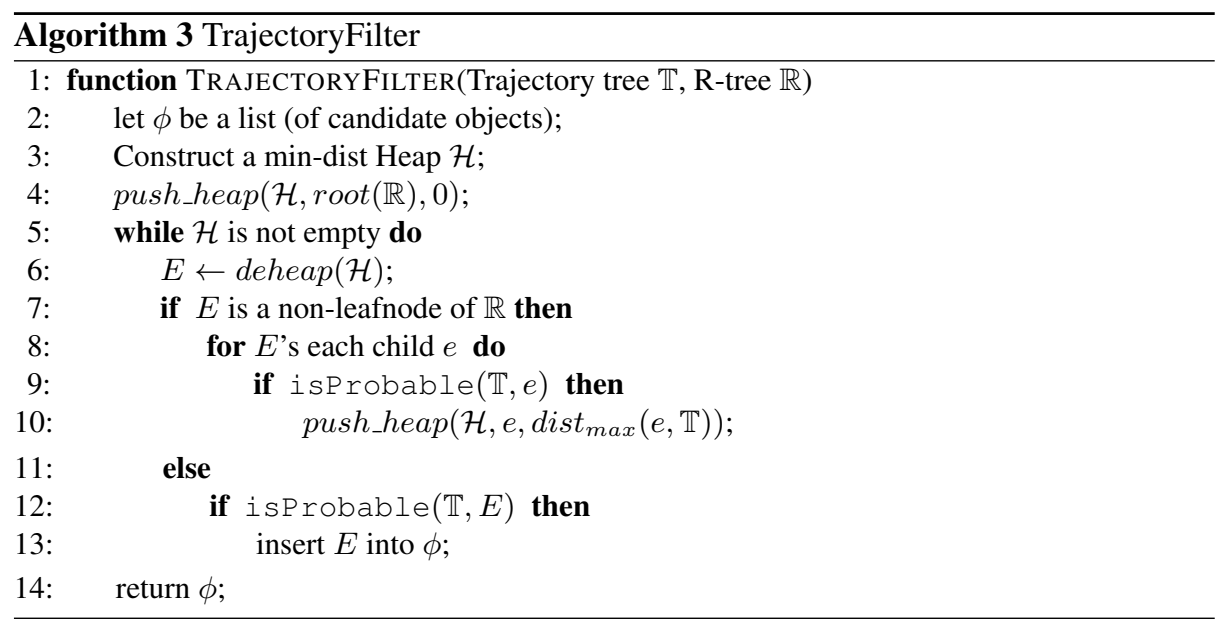

a trajectory tree node $T_{i}$ 's center. If $T_{i}$ is leaf-node, it is a line-segment. $T_{i}$ 's center is its mid-point. Otherwise, the center is $M B C\left(T_{i}\right)$ 's circle center. Then, the top element is popped to test if it/its children could be qualified to be the candidate objects. The process is repeated until the heap is empty.

To determine if an entry is qualified or not, we use Algorithm 4 . Let $T_{i}$ be a T's node and $G$ be $T_{i}$.Guard. $G$ is initialized in Step 4. Given an R-tree node $E$, if $T_{i} \subseteq H_{G}(E)$, then $\forall O_{j} \in E, O_{j}$ cannot be $T_{i}$ 's $P N N$ s. Thus, $\forall O_{j} \in E$ can be rejected. This helps pruning those unqualified objects in a higher index level.

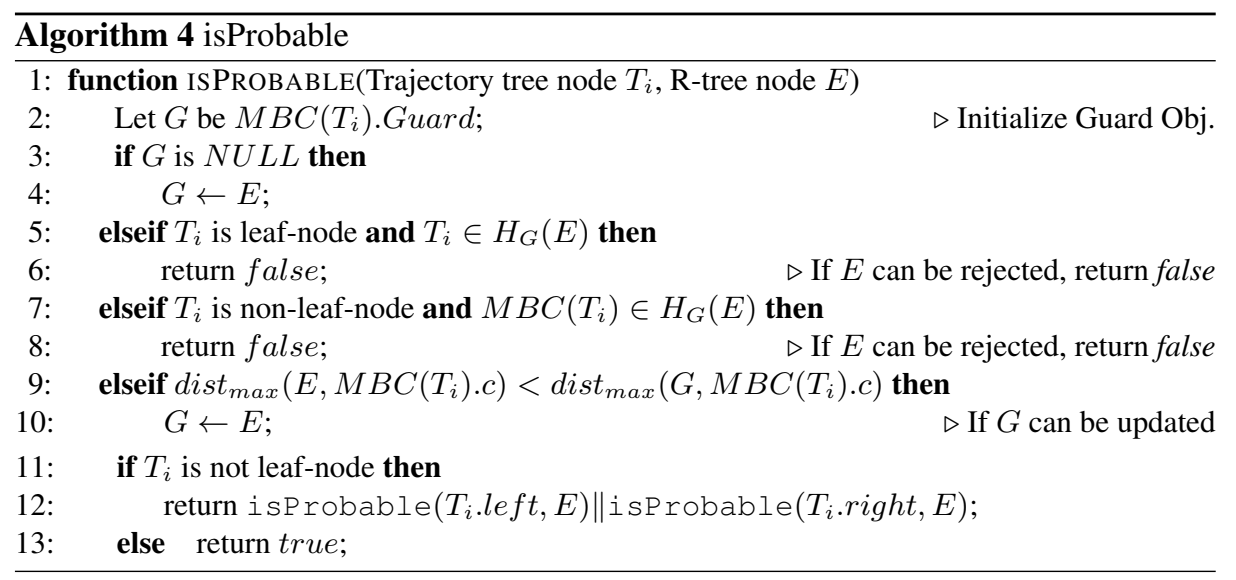

In order to check whether $E$ can be rejected, we consider two cases: (i) if $T_{i}$ is a leaf-node; (ii) if $T_{i}$ is a non-leaf-node.

(i) When $T_{i}$ is a leaf-node, we can draw a pruning bound to test whether $E$ is qualified. If we denote $\odot(c, G)$ as a circle centered at $c$ and internally tangent with object $G$, and $\odot(c, r)$ as a circle centered at $c$ with radius $r$, then:

$$
\odot(c, G)=\odot\left(c, \operatorname{dist}_{\max }(c, G)\right)
$$


The pruning bound is written as: $\odot(s, G) \cup \odot(e, G)$. The correctness is guaranteed by Lemma 1 .

Lemma 1. Given two imprecise objects $O_{i}, O_{j}$ and a line-segment $L(s, e), O_{j}$ can not be $p \in L$ 's $P N N$ if $O_{j}$ does not overlap with $\odot\left(s, O_{i}\right) \cup \odot\left(e, O_{i}\right)$.

Proof. To judge if $O_{j}$ is $L$ 's $P N N$, we first prove it is sufficient to check $L$ 's two end points $s$ and $e$. Then, we show how the pruning bound can be derived by $s$ and $e$.

Since $b_{i}(j)$ is a hyperbola half, it has at most two intersections with an arbitrary line. Thus, $H_{i}(j)$ is convex [15]. So, if $s$ and $e$ are in $H_{i}(j), p \in L$ must be in $H_{i}(j)$. It means if $O_{j}$ is not $s$ and $e$ 's $P N N$ given $O_{i}$, it is not a $P N N$ for all the points on $L$.

Next,

$$
\begin{aligned}
& s \in H_{i}(j) \Leftrightarrow \operatorname{dist}_{\max }\left(s, O_{i}\right)<\operatorname{dist}_{\min }\left(s, O_{j}\right) \\
& \left.\begin{array}{l}
\Leftrightarrow \odot\left(s, O_{i}\right) \cap O_{j}=\emptyset \\
\odot\left(e, O_{i}\right) \cap O_{j}=\emptyset
\end{array}\right\} \Leftrightarrow O_{j} \bigcap \odot\left(s, O_{i}\right) \cup \odot\left(e, O_{i}\right)=\emptyset
\end{aligned}
$$

So, the lemma is proved.

If another object $O_{j}$ does not overlap with the pruning bound defined by Lemma 1 , it can not be the $P N N$ of any $p \in L$, since $O_{i}$ will be always be closer. We also use Lemma 1 as the base to derive other pruning bounds in Section 6

(ii) When $T_{i}$ is a non-leaf-node, if $M B C\left(T_{i}\right) \in H_{G}(E)$, then $E$ can be rejected from candidates. Since $M B C\left(T_{i}\right) \in H_{G}(E), T_{i}$ must be in $H_{G}(E)$. In other words, Equation 6 is satisfied when the condition below is true:

$$
\operatorname{dist}_{\max }\left(M B C\left(T_{i}\right), G\right) \leq \operatorname{dist}_{\min }\left(M B C\left(T_{i}\right), E\right)
$$

Since $M B C\left(T_{i}\right)$ is a circle, Equation 6 can be rewritten as $(\odot . c$ and $\odot . r$ are $\odot$ 's center and radius):

$\operatorname{dist}_{\max }\left(M B C\left(T_{i}\right) \cdot c, G\right)+M B C\left(T_{i}\right) \cdot r \leq \operatorname{dist}_{\min }\left(M B C\left(T_{i}\right) \cdot c, E\right)-M B C\left(T_{i}\right) \cdot r$

\subsection{Segment Filter}

After the trajectory filtering step of $T P N N Q$, we get a set $\phi$ of candidates. Before passing $\phi$ to each line-segment $L_{i}$ in the refinement phase, we perform a simple filtering process to shrink $\phi$ into a smaller set $\phi_{i}$ for $L_{i}$. Notice that while deriving the trajectory tree $\mathbb{T}(\mathcal{T})$, we also derive an object called "Guard" for each node $T_{i}$. Then, for a $\mathcal{T}$ 's line-segment $L_{i}\left(s_{i}, e_{i}\right)$, we can reuse the "Guard" $O_{g}$ to build the pruning bound. According to Lemma 1 , the pruning bound is set to $\odot\left(s_{i}, O_{g}\right) \cup \odot\left(e_{i}, O_{g}\right)$. An example is shown in Figure 2(c), where the pruning bound for $L_{2}(h, t)$ is $\odot(h, d) \cup \odot(t, d)$. After that, we get $\phi_{i}$. Empirically, the pruned candidates set $\phi_{i}$ is much smaller than $\phi$.

\section{Trajectory Refinement Phase}

For trajectory $\mathcal{T}$, the refinement is done by applying Algorithm 5 Ternary Decomposition for each line-segment $L_{i} \in \mathcal{T}$. Essentially, Algorithm 5 is to construct a ternary tree $\Psi\left(L_{i}\right)$ for $L_{i}$. 


\subsection{Trajectory Refinement}

$\Psi$ is constructed in an iterative manner. At each iteration, we select two objects from the current candidate set $\phi_{c u r}$ as seeds to divide the current line-segment $L_{c u r}$ into two/three pieces.

To split $L_{i}$, we have to evaluate a feasible $u$-bisector, whose intersections with $L_{i}$ are turning points. Then, to find the $u$-bisector, we might have to try $\frac{C(C-1)}{2}$ pairs of objects, $C=\left|\phi_{i}\right|$. In fact, the object with minimum maximum distance to $L_{i}$, say $O_{1}$, must be one $P N N$. The correctness is shown in Lemma 2. Thus, it is often that the turning points on $L_{i}$ is derived by $O_{1}$ and another object among the $C$ candidates. So, in Algorithm 5, the candidates are sorted first.

Lemma 2. If $S=\left\{O_{1}, O_{2}, \ldots\right\}$ are sorted in the ascending order of the maximum distance to the line-segment $L$, then $O_{1} \in T P N N Q(L)$.

Proof. Suppose $p$ is a point of $L$, such that $\operatorname{dist}_{\max }\left(p, O_{1}\right)=\operatorname{dist}_{\max }\left(L, O_{1}\right)$. If $O_{1}$ is definitely one $P N N$ of $p \in L, O_{1}$ must be one $P N N$ of $L$. Thus, it is sufficient to show $O_{1} \in P N N Q(p)$.

To show $O_{1} \in P N N Q(p)$ is equivalent to prove $\operatorname{dist}_{\min }\left(p, O_{1}\right)<\operatorname{dist}_{\max }\left(p, O_{i}\right)$ $\left(O_{i} \in S\right)$. Then, it is sufficient to show $\operatorname{dist}_{\max }\left(p, O_{1}\right)<\operatorname{dist}_{\max }\left(p, O_{i}\right)\left(O_{i} \in S\right)$, as $\operatorname{dist}_{\min }\left(p, O_{1}\right)<\operatorname{dist}_{\max }\left(p, O_{1}\right)$.

Notice that $\operatorname{dist}_{\max }\left(p, O_{i}\right)$ must be no less than $\operatorname{dist}_{\max }\left(L, O_{i}\right)$. Then,

$$
\begin{aligned}
\operatorname{dist}_{\max }\left(p, O_{1}\right)=\operatorname{dist}_{\max }\left(L, O_{1}\right) & \leq \operatorname{dist}_{\max }\left(L, O_{i}\right)\left(O_{i} \in S\right) \\
& \leq \operatorname{dist}_{\max }\left(p, O_{i}\right)\left(O_{i} \in S\right)
\end{aligned}
$$

So, $O_{1}$ definitely belongs to $T P N N Q(L)$.

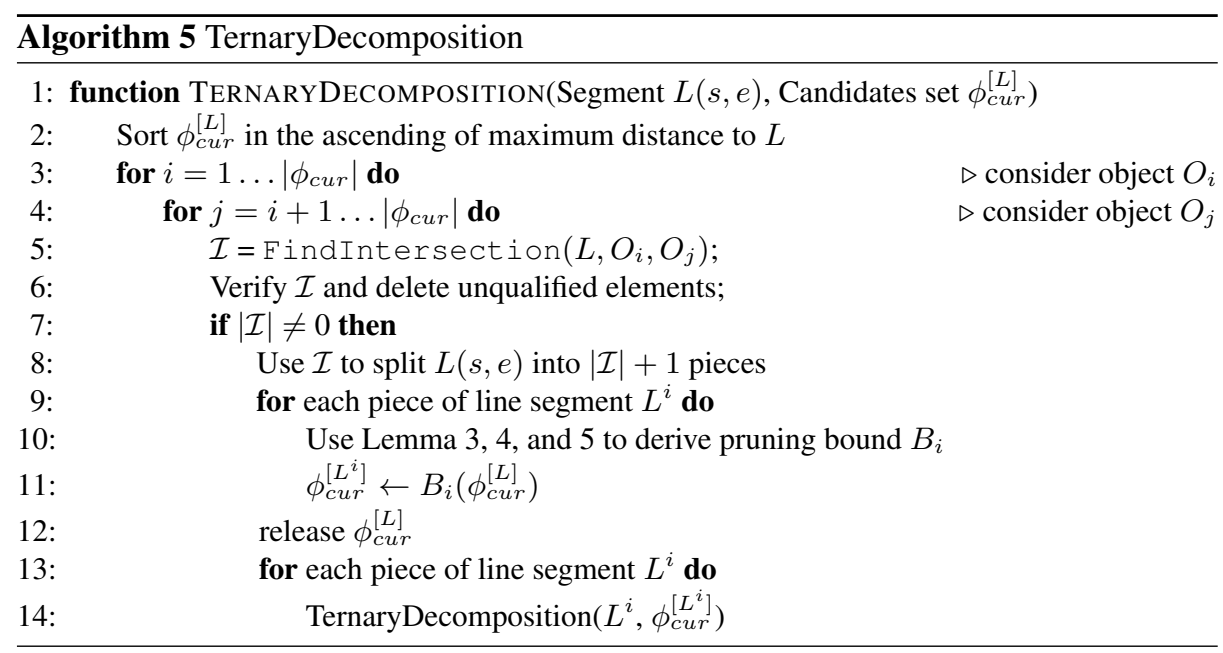

Then, $L_{c u r}$ is split into 2 (or 3) pieces ( or children). For $L_{c u r}$ 's children $L^{i}$, we derive a pruning bound $B_{i}$ for $L^{i}$ and select a subset of candidates from $\phi_{c u r}$, as shown in Step 9 to Step 12. 
Notice that for each leaf-node $L^{i}$ of the ternary tree $\Psi(L(s, e)), L^{i}$ 's two end points must be $s, e$, or the turning points on $L$. If we traverse $\Psi$ in the pre-order manner, any two successively visited leaf-nodes are the successively connected validity intervals in $L$. Suppose we have $m$ turning points, we would have $m+1$ validity intervals, which corresponds to $m+1 \Psi$ 's leaf-nodes.

Algorithm 5 stops when any pair of objects in $\phi_{\text {cur }}^{[L]}$ does not further split $L$. The complexity depends on the size of the turning points in the final answer. Recall the splitting process of Ternary Decomposition, a ternary tree node $T_{i}$ splits only if one or two intersections are found in $T_{i}$ 's line-segment. If no intersections found in its linesegment, $T_{i}$ becomes a leaf-node. Given the final answer containing $m$ turning points, there would be at most $2 m$ nodes in the ternary tree $\Psi(\mathcal{T})$. At least, there are $\lceil 1.5 m\rceil$ nodes. So, Algorithm 5 will be called $(1.5 m, 2 m]$ times. Step 5 is done in $\beta$ and Step 6 is in $O(\log C)$. If the candidate answers returned by Phase $I$ contains $C$ objects, the complexity of Phase II is $O\left(m C^{2}(\log C+\beta)\right)$. Next, we study how to derive the pruning bound $B_{i}$ mentioned in Step 11 .

\subsection{Pruning Bounds for Three Cases}

By a $u$-bisectors, a query line-segment could be divided into at most 3 sub-linesegments. The sub-line-segments fall into 3 categories according to their positions in half spaces. There are three types of sub-line-segments: Open Case, Pair Case, and Close Case, For example, in Figure 5. [ $\left.s_{d \vdash c}, s_{d \dashv c}\right]$ belongs to the pair case. Two examples of open case are $\left[h, s_{d \vdash c}\right]$ and $\left[s_{d \dashv c}, t\right]$. The Close Case means the line-segment is totally covered by a half-space. The three cases are formally described in Table 1 .

Table 1. Three cases for a line segment

\begin{tabular}{||l|l|l||}
\hline Case & Form & Position \\
\hline pair $\left[s_{i \vdash j}, s_{i \dashv j}\right]$ & $l \in V(i, j)$ \\
\hline open $\left[s, s_{i \vdash j}\right]$ or $\left[s_{i \dashv j}, e\right]$ & $l \in H_{i}(j)\left(\right.$ or $\left.l \in H_{j}(i)\right)(s(e)$ is the line-segment's start(end) point) \\
\hline close $\left[s_{i \vdash j}, s_{i \vdash j}^{\prime}\right]$ & $l \in H_{i}(j)$ and $s_{i \vdash j}, s_{i \vdash j}^{\prime} \in b_{i}(j)$ \\
\hline
\end{tabular}

For Pair Case and Open Case, we can derive two types of pruning bounds. Suppose the $u$-bisector between $O_{1}$ and $O_{2}$ split the query line-segment $[s, e]$ into sub-linesegments: $\left[s, s_{1 \vdash 2}\right],\left[s_{1 \vdash 2} s_{1 \dashv 2}\right]$, and $\left[s_{1 \dashv 2}, e\right]$, which are of Open Case, Pair Case, and Open Case, respectively. We shown the pruning bound derived for $\left[s, s_{1 \vdash 2}\right]$ and $\left[s_{1 \vdash 2} s_{1 \dashv 2}\right]$ in Figure 7 (a) and (b). The bounds are highlighted by shaded areas. The pruning bound of $\left[s_{1 \dashv 2}, e\right]$ is similar to Figure 7 (a), so it is omitted.

Close Case is a special case, when a line-segment has two intersections and totally inside one half-space, say $H_{i}(j)$. It could be represented by $\left[s_{i \vdash j}, s_{i \vdash j}^{\prime}\right]$, which means the two end-points are on the same $u$-bisector half $b_{i}(j)$. In this example, we known $\left[s_{i \vdash j}, s_{i \dashv j}^{\prime}\right]$ must be in $H_{i}(j)$, so $O_{j}$ cannot be the $P N N$ for each point inside. Next, we design their pruning bounds.

Lemma 3. (Pair Case) Suppose two imprecise objects $O_{i}$ and $O_{j}$, whose u-bisector $b_{i}(j)$ and $b_{j}(i)$ intersect with a straight line at $s_{i \vdash j}$ and $s_{i \dashv j}$. For another object $\forall O_{N} \in$ $O$, it cannot be $q \in\left[s_{i \vdash j} s_{i \dashv j}\right]$ 's $P N N$, if $O_{N}$ has no overlap with the pruning bound ○ $\left(s_{i \vdash j}, O_{i}\right) \cup \odot\left(s_{i \dashv j}, O_{j}\right) \bigcap \odot\left(s_{i \vdash j}, O_{j}\right) \cup \odot\left(s_{i \dashv j}, O_{i}\right)$. 
Proof. $\forall p \in\left[s_{i \vdash j} s_{i \dashv j}\right]$, both $O_{i}$ and $O_{j}$ have chances to be $p$ 's PNN. According to Lemma 1 a new object $O_{N}$ cannot be $O_{i}$ or $O_{j}$ 's nearest neighbor if

$$
O_{N} \bigcap\left(\odot\left(s_{i \vdash j}, O_{i}\right) \cup \odot\left(s_{i \dashv j}, O_{i}\right)\right)=\emptyset, \text { or } O_{N} \bigcap\left(\odot\left(s_{i \vdash j}, O_{j}\right) \cup \odot\left(s_{i \dashv j}, O_{j}\right)\right)=\emptyset
$$

So, the pruning bound is:

$$
\text { ๑ }\left(s_{i \vdash j}, O_{i}\right) \cup \odot\left(s_{i \dashv j}, O_{i}\right) \bigcap \odot\left(s_{i \vdash j}, O_{j}\right) \cup \odot\left(s_{i \dashv j}, O_{j}\right)
$$

Lemma 4. (Open Case) Given a line-segment $\left[s, s_{i \vdash j}\right]$, for other objects $\forall O_{N} \in O$, it cannot be query point $q \in\left[s, s_{i \vdash j}\right]$ 's nearest neighbor, if $O_{N}$ has no overlap with the $\odot\left(s, O_{i}\right) \cup \odot\left(s_{i \vdash j}, O_{i}\right)$.

Lemma 5. (Close Case) Given two split points $s_{i \vdash j}$ and $s_{i \vdash j}^{\prime}$, the pruning bound for $\left[s_{i \vdash j}, s_{i \vdash j}^{\prime}\right]$ is $\odot\left(s_{i \vdash j}, O_{i}\right) \cup \odot\left(s_{i \vdash j}^{\prime}, O_{i}\right)$.

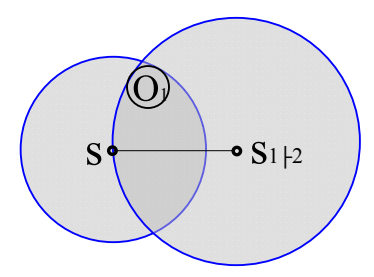

(a) Open Case

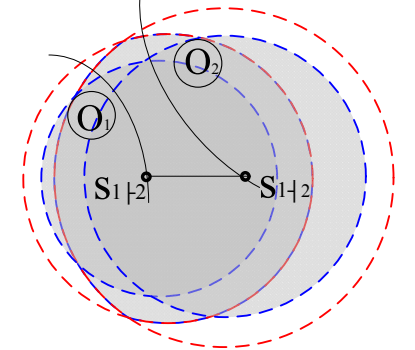

(b) Pair Case

Fig. 7. Open Case and Pair Case

Since the proofs of Lemma 5 and Lemma 4 can be easily derived from Lemma 1 , they are omitted due to page limitation. The Pair Case could also be considered as the overlap of two Open Cases. For example, a Pair Case $\left[s_{i \vdash j}, s_{i \dashv j}\right]$ is equivalent to the overlap part of $\left[s, s_{i \dashv j}\right]$ and $\left[s_{i \vdash j}, e\right]$. Also, the Close Case could be viewed as the overlap of $\left[s, s_{i \dashv j}^{\prime}\right]$ and $\left[s_{i \vdash j}, e\right]$. The three cases and their combinations could cover all the cases for each piece (validity interval) of the line segment. After $\Psi$ 's construction is done, we can view the pruning bound of a validity interval. It is the intersection of all its ascender nodes' pruning bounds in the ternary tree $\Psi$.

\section{Experimental Results}

Section 7.1 describes settings. We adopt a metric to measure to quality of results in Section 7.2 Section 7.3 discusses the results.

\subsection{Setup}

Queries The query trajectories are generated by Brinkhoff's network-based mobile data generator ${ }^{4}$ The trajectory represents movements over the road-network of Oldenburg city in Germany. We normalize them into $10 \mathrm{k} \times 10 \mathrm{k}$ space. By default, the length of trajectory is 500 units. Each reported value is the average of 20 trajectory query runs.

Imprecise Objects We use four real datasets of geographical objects in Germany and US 5 , namely germany, LB, stream and block with 30k, 50K, 199K, 550k spatial objects,

\footnotetext{
${ }^{4}$ http://iapg.jade-hs.de/personen/brinkhoff/generator/

${ }^{5}$ http://www.rtreeportal.org/
} 
respectively. We use stream as the default dataset. We construct the $M B C$ for each object thus get 4 datasets with circular imprecise regions. Datasets are normalized to the same domain as queries. To index imprecise regions, we use a packed $\mathrm{R}^{*}$-tree [16]. The page size of R-tree is set to $4 \mathrm{k}$-byte, and the fanout is 50 . The entire R-tree is accommodated in the main memory.

For the turning points calculation, we call GSL Library ${ }^{6}$ to get the analytical solution. All our programs were implemented in $\mathrm{C}++$ and tested on a Core2 Duo $2.83 \mathrm{GHz}$ PC.

\subsection{Quality Metric}

To measure the accuracy of a query result, we adopt a Error function based on the Jaccard Distance [17], which is used in comparing the similarity between two sets. Recall the definition of TPNNQ the query result is a set of tuples $\left\{\left\langle T_{i}, R_{i}\right\rangle\right\}$. It can be transformed into the $P N N$ s for every point on the query trajectory. Formally, the result is $\{\langle q, P N N Q(q)\rangle\}_{q \in \mathcal{T}}$. Let $R^{*}(q)$ be the optimal solution for the point $q$, where $R^{*}(q)=P N N Q(q)$. We use $R^{A}(q)$ to represent the $P N N$ s derived for the point $q$ in algorithm $A$. Then, the Error for algorithm $A$ on query $\mathcal{T}$ is:

$$
\operatorname{Error}(\mathcal{T}, A)=\frac{1}{|\mathcal{T}|} \int_{q \in \mathcal{T}} 1-\frac{R^{*}(q) \cap R^{A}(q)}{R^{*}(q) \cup R^{A}(q)} d q
$$

$|\mathcal{T}|$ is the total length of trajectory $\mathcal{T}$. If $\mathcal{T}$ is represented by a set of line-segments $\mathcal{T}=\left\{L_{i}\right\}_{i=1}^{t}$, the total length $|\mathcal{T}|=\sum_{i=1}^{t}\left|L_{i}\right|$.

Equation 8 captures the effect of false positives and false negatives as well. There is a false positive when $R^{A}(q)$ contains an extra item not found in $R^{*}(q)$. There is a false negative when an item of $R^{*}(q)$ is missing from $R^{A}(q)$. For a perfect method with no false positives and false negatives, the two terms $R^{*}(q)$ and $R^{A}(q)$ are the same, so the integration value is 0 .

In summary, the error score is a value between 0 and 1. The smaller an Error score is, the more accurate the result is. On the other hand, if a method has many extra or missing results, then it obtains a high Error.

\subsection{Performance Evaluation}

The query performance is evaluated by two metrics: efficiency and quality. The efficiency is measured by counting the clock time. The quality is measured by the error score. We compare four methods: Basic, Sample, TP-S, and TP-TS. The suffixes $T$ and $S$ refer to Trajectory Filter and Segment Filter, respectively. Basic does not use any filter; TP-S does not use Trajectory Filter; TP-TS (Algorithm2) uses all the filtering and refinement techniques. Sample draws a set of uniform sampling points $\{q\}$ from $\mathcal{T}$. Then, for all $q$, $P N N Q(q)$ is evaluated. The sampling interval, denoted by $\epsilon$, is set to 0.1 unit.

Query Efficiency $\boldsymbol{T}_{\boldsymbol{q}}$ From Figure 8, the Basic method is the slowest method among all the four, since it elaborates all the possible pairs of objects for turning points (but most of them do not contribute to validity intervals). For the second slowest Sample, we analyze it later.

\footnotetext{
${ }^{6}$ http://www.gnu.org/software/gsl/

7 The sampling rate is reasonably high regarding to the trajectory's default length. More details about sampling rates are discussed later.
} 


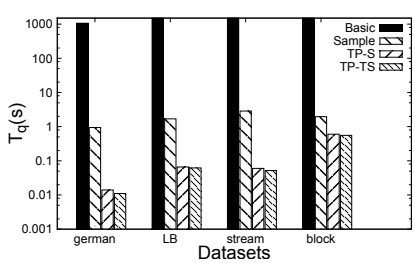

Fig. 8. $T_{q}(\mathrm{~s}) \quad$ vs. Datasets

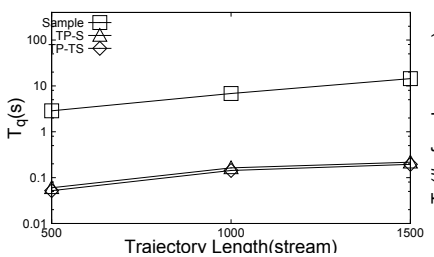

Fig. 11. $T_{q}$ vs. Query Length

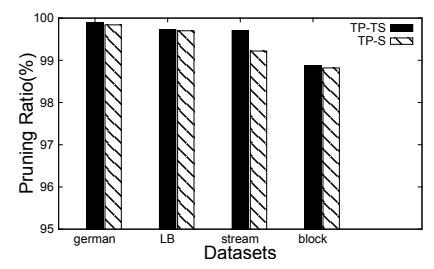

Fig. 9. Pruning Ratio vs. Datasets

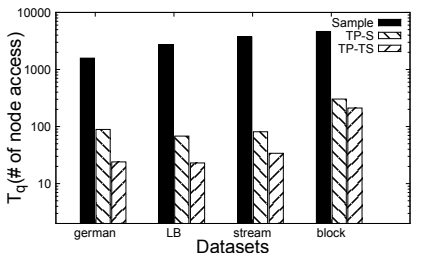

Fig. 12. $T_{q}$ (\# of node access) vs. Datasets

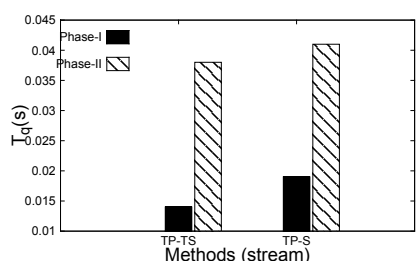

Fig. 10. $T_{q}$ 's breakdown

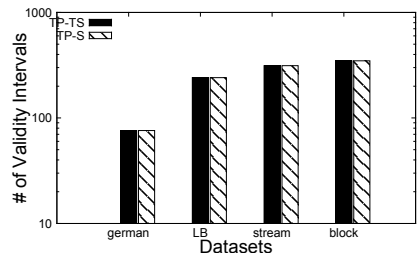

Fig. 13. $\sharp$ of Validity Intervals vs. Datasets

The other two methods have significant improvement over Sample and Basic. One reason is because of the effectiveness of the pruning techniques, as shown in Figure 9 For all the real datasets, the pruning ratio are as high as $98.8 \%$. TP-S is less efficient, because some candidates shared by different line-segments in trajectory will be fetched multiple times. This drawback is overcome by TP-TS.

To get a clearer picture about the efficiency of our framework, we measure the time costs for Phase I and Phase II in Figure 10. TP-TS is faster in both phases. In Phase I, the combined R-tree traversal in TP-TS saves plenty of extra node access, compared to TP-S. The number of node access is shown in Figure 12. In Phase II, TP-TS is faster, since it has fewer candidates to handle. This observation is also consistent with the fact that TP-TS has a higher pruning ratio, shown in Figure 9

We also test the query efficiency by varying the query length in Figure 11. The Sample method is slower than others at least one order of magnitude. The costs of other two methods increase slowly w.r.t. the query length.

TP-TS vs. Sample Sample method is a straightforward solution to approximate the $T P N N Q$ answer. However, this solution suffers from the extensive R-tree traversal$\mathrm{s}$, since every sampling point $q$ requires accessing of R-tree. As shown in Figure 12 , Sample incurs at least more than one order of magnitude node access than our method.

On the other hand, Sample could incur false negatives, even with a large sampling rate. Because Sample only considers query points sampled on the trajectory, whereas $T P N N Q$ is for all the points in $\mathcal{T}$. To calculate Sample's error score, we have to infer the $P N N$ s for a point $q \in \mathcal{T}$ not being sampled, as required by Equation 8 With limited sampled answers, $q$ 's $P N N$ s can only be "guessed" by using its closest sampling point $p$. In other words, $P N N Q(q)$ has to be substituted with $P N N Q(p)$.

The efficiency is reflected in Figure 14 , where the sampling interval $\epsilon$ is varied from 0.01 to 10 . We can observe that TP-TS outperforms Sample in most of the cases. Sample is faster only when $\epsilon$ is very large (e.g. equal to 10 units). Then, is it good if large $\epsilon$ is 
used? The answer is NO. In Table 15, when "Sample, $\epsilon=10$, block", the error score of Sample is as high as 0.443 !

We demonstrate the error score of Sample and TP-TS in Table 15. Since TP-TS evaluate the exact answer, the error is always 0. The error of Sample is small when $\epsilon$ is small, (e.g. equal to 0.01, block). However, the query time of that case is 100 times slower than TP-TS. We would like to emphasize that even the error score is empirically tested to be 0 over large sampling rates, there is no theoretical guarantee for the Sample to contain 0 false negative.

We also test the error score of simplifying the imprecise regions into precise points, as mentioned in the introduction. For german dataset, the error is as high as 0.76 ! Thus, the simplified solution could be harmful for applications such as safety sailing.

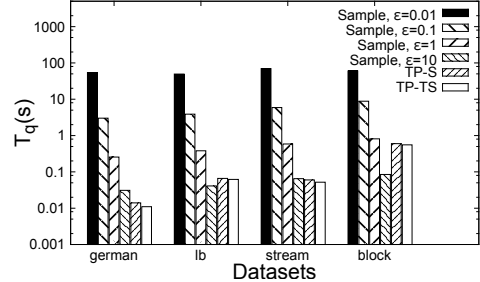

Fig. 14. TP-TS vs. Sample $\left(T_{q}\right)$

\begin{tabular}{||c|l|l|l|l|l|}
\hline \multirow{2}{*}{ Datasets } & \multicolumn{4}{|c|}{ Sample } & \multirow{2}{*}{ TP-TS } \\
\cline { 2 - 5 } & $\epsilon=0.01$ & $\epsilon=0.1$ & $\epsilon=1$ & $\epsilon=10$ & \\
\hline german & 0.00340 & 0.00457 & 0.01528 & 0.12310 & 0 \\
\hline LB & 0.00005 & 0.00029 & 0.00257 & 0.02672 & 0 \\
\hline stream & 0.00059 & 0.00090 & 0.00298 & 0.03962 & 0 \\
\hline block & 0.01872 & 0.02541 & 0.08516 & 0.44310 & 0 \\
\hline \hline
\end{tabular}

Fig. 15. TP-TS vs. Sample(Error)

Analysis of TPNN Observed from Figure 13 , the number of validity intervals increases with the size of the datasets. TP-S and TP-TS have the same number of validity intervals, which is as expected.

In summary, we have shown that TP-TS is much more efficient than Basic, Sample, and TP-S methods. It also achieves much better quality than Sample method.

\section{Conclusion}

In this paper, we study the problem of trajectory query over imprecise data. To tackle the low quality and inefficiency in simplified methods, we study the geometric properties of the $u$-bisector. Based on that, we design several novel filters to support our algorithm. Extensive experiments show that our method can efficiently evaluate the TPNNQ with high quality.

The geometric theories studied in this paper has no limitations in the dimensionality and shape of imprecise regions. In future, we would like to evaluate the algorithm's performance in multi-dimensional space with different shaped imprecise regions. We would also extend our work to support variants queries like k-PNN query, etc.

\section{Acknowledgment}

Reynold Cheng and Xike Xie were supported by the Research Grants Council of Hong Kong (GRF Projects 513508, 711309E, 711110). Man Lung Yiu was supported by ICRG grants A-PJ79 and G-U807 from the Hong Kong Polytechnic University. We would like to thank the anonymous reviewers for their insightful comments. 


\section{References}

1. Y. Tao, D. Papadias, and Q. Shen, "Continuous nearest neighbor search," in VLDB, 2002.

2. U. S. C. Guard, "Announcement of 2011 international ice patrol services," http://www.uscg. mil/lantarea/iip/docs/AOS_2011.pdf

3. L. Jesse, R. Janet, G. Edward, and V. Lee, "Effects of habitat on gps collar performance : using data screening to reduce location error," in Journal of applied ecology, 2007.

4. K. Park, H. Choo, and P. Valduriez, "A scalable energy-efficient continuous nearest neighbor search in wireless broadcast systems," Wireless Networks, 2010.

5. R. Cheng, X. Xie, M. L. Yiu, J. Chen, and L. Sun, "Uv-diagram: A voronoi diagram for uncertain data," in ICDE, 2010.

6. X. Lian and L. Chen, "Efficient processing of probabilistic reverse nearest neighbor queries over uncertain data." in VLDBJ, 2009.

7. M. A. Cheema, X. Lin, W. Wang, W. Zhang, and J. Pei, "Probabilistic reverse nearest neighbor queries on uncertain data." in TKDE, 2010.

8. J. Chen, R. Cheng, M. Mokbel, and C. Chow, "Scalable processing of snapshot and continuous nearest-neighbor queries over one-dimensional uncertain data," in VLDBJ, 2009.

9. G. Trajcevski, R. Tamassia, H. Ding, P. Scheuermann, and I. F. Cruz, "Continuous probabilistic nearest-neighbor queries for uncertain trajectories," in EDBT, 2009, pp. 874-885.

10. K. Zheng, G. P. C. Fung, and X. Zhou, "K-nearest neighbor search for fuzzy objects," in SIGMOD, 2010.

11. Z. Song and N. Roussopoulos, "K-nearest neighbor search for moving query point," in SSTD, 2001.

12. Z. Baihua and L. Dik, "Semantic caching in location-dependent query processing," in Advances in Spatial and Temporal Databases, 2001.

13. J. Zhang, M. Zhu, D. Papadias, Y. Tao, and D. L. Lee, "Location-based spatial queries." in SIGMOD, 2003.

14. R. Cheng, D. V. Kalashnikov, and S. Prabhakar, "Querying imprecise data in moving object environments," TKDE, vol. 16, no. 9, 2004.

15. S. Boyd and L. Vandenberghe, Convex Optimization. Cambridge University Press, 2004.

16. M.Hadjieleftheriou, "Spatial index library version 0.44.2b." [Online]. Available: http: //u-foria.org/marioh/spatialindex/index.html

17. P.-N. Tan, M. Steinbach, and V. Kumar, "Introduction to data mining," 2006.

\section{A Appendix}

\section{A.1 Intersection of a hyperbola and a straight line}

Given a hyperbola $h_{1}$ and a straight line $l_{1}$, they could have 0,1 , or 2 intersection points, which is the roots of the following:

$$
\left\{\begin{array}{l}
h_{1}: \frac{x^{2}}{a_{1}^{2}}-\frac{y^{2}}{b_{1}^{2}}=1 \\
l_{1}: a_{2} x+b_{2} y+c_{2}=0
\end{array}\right.
$$

By solving Equation 9, we can have:

$$
\left\{\begin{array}{l}
x=\frac{-a_{1}^{2} a_{2} c_{2} \pm \sqrt{-a_{1}^{2} b_{1}^{2} b_{2}^{2}\left(a_{1}^{2} a_{2}^{2}-b_{1}^{2} b_{2}^{2}-c_{2}^{2}\right)}}{a_{1}^{2} a_{2}^{2}-b_{1}^{2} b_{2}^{2}} \\
y=\frac{-a_{2} \pm \sqrt{a_{1}^{2} b_{1}^{2} b_{2}^{2}\left(-a_{1}^{2} a_{2}^{2}+b_{1}^{2} b_{2}^{2}+c_{2}^{2}\right)}-b_{1}^{2} b_{2}^{2} c_{2}}{b_{2}\left(b_{1}^{2} b_{2}^{2}-a_{1}^{2} a_{2}^{2}\right)}
\end{array}\right.
$$

$$
\text { where }\left\{\begin{array}{l}
a_{1}^{2} a_{2}^{2}-b_{1}^{2} b_{2}^{2} \neq 0 \\
b_{2} \neq 0 \\
a_{1} b_{1} \neq 0
\end{array}\right.
$$

Notice that if any of the three pre-conditions in Equation 11 is not satisfied, there should be no intersection point for the given curve and line. 